\title{
BAND SURGERY ON KNOTS AND LINKS
}

\section{TAIZO KANENOBU}

\begin{tabular}{|c|l|}
\hline Citation & OCAMI Preprint Series \\
\hline Issue Date & 2009 \\
\hline Type & Preprint \\
\hline Textversion & Author \\
\hline Rights & For personal use only. No other uses without permission. \\
\hline Relation & $\begin{array}{l}\text { Preprint of an article submitted for consideration in Journal of Knot Theory and } \\
\text { Its Ramifications } @ \text { C 2010 World Scientific Publishing Company. } \\
\text { https://www.worldscientific.com/worldscinet/jktr . }\end{array}$ \\
\hline Is version of & $\underline{\text { https://doi.org/10.1142/S0218216510008522 }}$ \\
\hline
\end{tabular}

From: Osaka City University Advanced Mathematical Institute http://www.sci.osaka-cu.ac.jp/OCAMI/publication/preprint/preprint.html 


\title{
BAND SURGERY ON KNOTS AND LINKS
}

\author{
TAIZO KANENOBU \\ Department of Mathematics, Osaka City University \\ Sugimoto, Sumiyoshi-ku, Osaka 558-8585, Japan \\ kanenobu@sci.osaka-cu.ac.jp
}

\begin{abstract}
We give some relationships of the Jones and Q polynomials between two links which are related by a band surgery. Then we consider two applications: The first one is to an evaluation of the ribbon-fusion number, the least fusion number of a ribbon knot. The second one is to DNA knot theory, helping us to understand the action of the Xer site-specific recombination at psi site.
\end{abstract}

Keywords: Knot; Link; Band surgery; Jones polynomial; Q polynomial; ribbon-fusion number; DNA knot; Xer recombination.

Mathematics Subject Classification 2000: 57M25, 57M27

\section{Introduction}

Let $L$ be an oriented link, and $b: I \times I \rightarrow S^{3}$ an embedding such that $b(I \times I) \cap$ $L=b(I \times \partial I)$, where $I$ is a closed interval. Let $L^{\prime}=(L-b(I \times \partial I)) \cup b(\partial I \times I)$, which is another link. If $L^{\prime}$ has the orientation compatible with the orientation of $L-b(I \times I) \cap L$ and $b(\partial I \times I)$, then $L^{\prime}$ is called the link obtained from $L$ by the band surgery along the band $b$. Then there is a relation between the signatures of $L$ and $L^{\prime}$ due to Murasugi; see Eq. (2.2). In this paper, we give further relationships in terms of the Jones polynomial (Theorem 2.2) and the Q polynomial (Theorem 3.1). Then we apply these relations in two ways: The first application is to estimate the ribbon-fusion number of a ribbon knot. A knot is a ribbon knot if it is a knot obtained from a trivial $(m+1)$-component link by doing band surgery along $m$ bands for some $m$. We call the least number of such $m$ the ribbon-fusion number. There is an estimation for this number due to Sakuma, which is given in terms of the Nakanishi index (Proposition 4.2). Using the above-mentioned relationships we deduce Theorems 4.3 and 4.4, which can give a sharper estimation (Examples 4.6, 4.7).

The second application is to consider a problem whether a given knot with $(2 n+1)$ crossings is related to a $(2,2 n)$ torus link or not by a band surgery, which was brought from the study of a DNA site-specific recombination. More precisely, Bath, Sherratt, and Colloms [1] have shown that the action of the Xer site-specific 
recombination at $p s i$ site is the change from a $(2,2 n)$ torus link to a $(2 n+1)$-crossing knot by a band surgery. So characterizing such change is an important problem. Applying Theorems 2.2 and 3.1, we will show the 7 crossing knots $7_{3}, 7_{6}$ cannot be obtained from a $(2,6)$ torus link (Proposition 5.4), and the 9 crossing knots $9_{15}$, $9_{17}, 9_{31}$ cannot be obtained from a $(2,8)$ torus link (Propositions 5.6 and 5.7).

Notation. For knots with up to 10 crossings we use Rolfsen notation [23, Appendix $\mathrm{C}]$.

\section{The Jones Polynomial}

In this section, we give a relationship of the Jones polynomials of two links that are related by a band surgery. Before that we review a classical result for the signature of these links due to Murasugi. Let $L_{+}, L_{-}, L_{0}$ be three links that are identical except near one point where they are as in Fig. 1 ; we call $\left(L_{+}, L_{-}, L_{0}\right)$ a skein triple. Then Murasugi [19, Lemma 7.1] has shown:

$$
\left|\sigma\left(L_{ \pm}\right)-\sigma\left(L_{0}\right)\right| \leq 1
$$

Since we may consider the link $L_{+}$or $L_{-}$is obtained from $L_{0}$ by a band surgery, and vice versa, two links $L$ and $L^{\prime}$ which are related by a band surgery satisfy:

$$
\left|\sigma(L)-\sigma\left(L^{\prime}\right)\right| \leq 1 \text {. }
$$

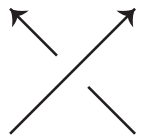

$L_{+}$

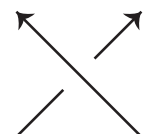

$L_{-}$

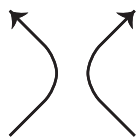

$L_{0}$

Fig. 1. A skein triple.

The Jones polynomial $V(L ; t) \in \boldsymbol{Z}\left[t^{ \pm 1 / 2}\right]$ [8], is an invariant of the isotopy type of an oriented link $L$, which is defined by the following formulas:

$$
\begin{gathered}
V(U ; t)=1 \\
t^{-1} V\left(L_{+} ; t\right)-t V\left(L_{-} ; t\right)=\left(t^{1 / 2}-t^{-1 / 2}\right) V\left(L_{0} ; t\right),
\end{gathered}
$$

where $U$ is the unknot and $\left(L_{+}, L_{-}, L_{0}\right)$ is a skein triple.

We put $\omega=e^{i \pi / 3}$. For a knot $K$, Lickorish and Millett [14, Theorem 3] have shown:

$$
V(L ; \omega)= \pm i^{c(L)-1}(i \sqrt{3})^{d},
$$

where $c(L)$ is the number of the components of $L, d=\operatorname{dim} H_{1}\left(\Sigma(L) ; \boldsymbol{Z}_{3}\right)$ with $\Sigma(L)$ the double cover of $S^{3}$ branched over $L$; cf. [15]. Note that $V(L ; \omega)$ means the value of $V(L ; t)$ at $t^{1 / 2}=e^{i \pi / 6}$, whence $t^{1 / 2}-t^{-1 / 2}=i$. 
The following lemma is due to Miyazawa [17].

\section{Lemma 2.1.}

$$
\frac{V\left(L_{+} ; \omega\right)}{V\left(L_{-} ; \omega\right)} \in\left\{ \pm 1, i \sqrt{3}^{ \pm 1}\right\}
$$

Proof. For the skein triple $\left(L_{+}, L_{-}, L_{0}\right)$, we consider another oriented link $L_{\infty}$ which is one of the diagram of Fig. 2, the choice being (i) if $c\left(L_{+}\right)<c\left(L_{0}\right)$ and (ii) if $c\left(L_{+}\right)>c\left(L_{0}\right)$.

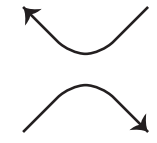

(i)

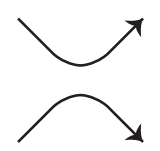

(ii)

Fig. 2. Two choices of the oriented link $L_{\infty}$.

Then by [2, Theorem 2] for the case (i) we have

$$
V\left(L_{+} ; t\right)-t V\left(L_{-} ; t\right)+t^{3 \lambda}(t-1) V\left(L_{\infty} ; t\right)=0,
$$

where $\lambda$ is the linking number of the right-hand component of $L_{0}$ in Fig. 1 with the remainder of $L_{0}$, and for the case (ii) we have

$$
V\left(L_{+} ; t\right)-t V\left(L_{-} ; t\right)+t^{3\left(\mu-\frac{1}{2}\right)}(t-1) V\left(L_{\infty} ; t\right)=0
$$

where $\mu$ is the linking number of the botttom-right and top-left component $L_{+}$in in Fig. 1 with the remainder of $L_{+}$.

We consider the case (i). Putting $t=\omega$ in (2.7), we have

$$
x-\omega+(-1)^{\lambda}(\omega-1) y=0,
$$

where $x=V\left(L_{+} ; \omega\right) / V\left(L_{-} ; \omega\right)$ and $y=V\left(L_{\infty} ; \omega\right) / V\left(L_{-} ; \omega\right)$. Then by Eq. $(2.5)$ there are four cases:
(a) $(x, y)=(\alpha, \beta)$;
(b) $(x, y)=(\alpha, \beta i)$;
(c) $(x, y)=(\alpha i, \beta)$;
(d) $(x, y)=(\alpha i, \beta i)$,

where $\alpha, \beta$ are real numbers. For the case (a), we have $\alpha=1, \beta=(-1)^{\lambda}$; for the case (b), we have $\alpha=-1, \beta=(-1)^{\lambda+1} \sqrt{3}$; for the case (c), we have $\alpha=\sqrt{3}$, $\beta=(-1)^{\lambda+1}$; for the case (d), we have $\alpha=\sqrt{3}^{-1}, \beta=(-1)^{\lambda+1} \sqrt{3}^{-1}$, obtaining the result. 
For the case (ii) we can prove similarly.

Theorem 2.2. Let $L$ and $L^{\prime}$ be two links related with a band surgery such that $c(L)<c\left(L^{\prime}\right)$. Then

$$
\frac{V(L ; \omega)}{V\left(L^{\prime} ; \omega\right)} \in\left\{ \pm i,-\sqrt{3}^{ \pm 1}\right\}
$$

Proof. From the condition there is a skein triple $\left(L_{+}, L_{-}, L_{0}\right)$ such that $L_{+}$and $L_{0}$ are isotopic to $L$ and $L^{\prime}$, respectively. Put $x=V\left(L_{+} ; \omega\right) / V\left(L_{-} ; \omega\right)$ and $z=$ $V\left(L_{0} ; \omega\right) / V\left(L_{-} ; \omega\right)$. Then by Eq. (2.4), we have

$$
\omega^{-1} x-\omega=i z
$$

and so

$$
\frac{V(L ; \omega)}{V\left(L^{\prime} ; \omega\right)}=\frac{x}{z}=\frac{i x}{\omega^{-1} x-\omega} .
$$

By Lemma 2.1 we obtain (2.2).

By using Eq. (2.5), Theorem 2.2 immediately implies the following.

Corollary 2.3. Suppose that a knot $K$ is obtained from a 2-component link $L$ by a band surgery. Then

$$
V(K ; \omega) \in \begin{cases}\{ \pm 1,-i \sqrt{3} \epsilon\} & \text { if } V(L ; \omega)=i \epsilon \\ \{-\epsilon, \pm i \sqrt{3},-3 \epsilon\} & \text { if } V(L ; \omega)=\sqrt{3} \epsilon,\end{cases}
$$

where $\epsilon= \pm 1$.

\section{The $Q$ Polynomial}

In this section, we give a relationship of the $\mathrm{Q}$ polynomials of two links that are related by a band surgery. The $Q$ polynomial $Q(L ; z) \in \boldsymbol{Z}\left[z^{ \pm 1}\right][4,6]$ is an invariant of the isotopy type of an unoriented link $L$, which is defined by the following formulas:

$$
\begin{gathered}
Q(U ; z)=1 ; \\
Q\left(L_{+} ; z\right)+Q\left(L_{-} ; z\right)=z\left(Q\left(L_{0} ; z\right)+Q\left(L_{\infty} ; z\right)\right),
\end{gathered}
$$

where $U$ is the unknot and $L_{+}, L_{-}, L_{0}, L_{\infty}$ are four unoriented links that are identical except near one point where they are as in Fig. 3. We call $\left(L_{+}, L_{-}, L_{0}, L_{\infty}\right)$ an unoriented skein quadruple.

We put $\rho(K)=Q(K ;(\sqrt{5}-1) / 2))$. For a knot $K$, Jones [9] has shown:

$$
\rho(K)= \pm \sqrt{5}^{r}
$$

where $r=\operatorname{dim} H_{1}\left(\Sigma(K) ; \boldsymbol{Z}_{5}\right)$ with $\Sigma(K)$ the double cover of $S^{3}$ branched over $K$. Furthermore, Rong [24] deduced some information on the values $\rho\left(L_{-}\right) / \rho\left(L_{\infty}\right)$, 


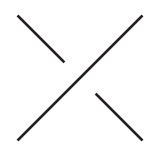

$L_{+}$

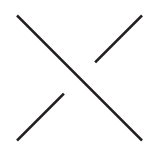

$L_{-}$

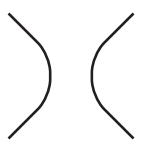

$L_{0}$

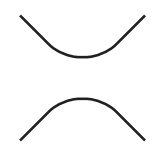

$L_{\infty}$

Fig. 3. An unoriented skein quadruple.

$\rho\left(L_{0}\right) / \rho\left(L_{\infty}\right), \quad \rho\left(L_{+}\right) / \rho\left(L_{\infty}\right)$, where $\left(L_{+}, L_{-}, L_{0}, L_{\infty}\right)$ is an unoriented skein quadruple. Using these values, we have the following, which is analogous to a criterion on the unknotting number of a knot due to Stoimenow [25, Theorem 4.1]; cf. [10].

Theorem 3.1. If two links $L$ and $L^{\prime}$ are related by a band surgery, then

$$
\rho(L) / \rho\left(L^{\prime}\right) \in\left\{ \pm 1, \sqrt{5}^{ \pm 1}\right\} .
$$

Proof. From the condition there is an unoriented skein quadruple $\left(L_{+}, L_{-}, L_{0}, L_{\infty}\right)$ such that $L_{0}$ and $L_{\infty}$ are isotopic to $L$ and $L^{\prime}$, respectively. Then from the proof of Theorem 2 in [24], we have $\rho\left(L_{0}\right) / \rho\left(L_{\infty}\right) \in\left\{ \pm 1, \sqrt{5}^{ \pm 1}\right\}$, obtaining the result.

By using (3.3), Theorem 3.1 immediately implies the following.

Corollary 3.2. Suppose that a knot $K$ is obtained from a link $L$ by a band surgery. Then

$$
\rho(K) \in \begin{cases}\{ \pm 1, \sqrt{5} \epsilon\} & \text { if } \rho(L)=\epsilon \\ \{1, \pm \sqrt{5}, 5\} & \text { if } \rho(L)=\sqrt{5}\end{cases}
$$

where $\epsilon= \pm 1$.

\section{The Ribbon-Fusion Number of a Ribbon Knot}

In this section, we apply the theorems given in the previous sections to an evaluation of the ribbon-fusion number of a ribbon knot. A knot is said to be a ribbon knot of $m$-fusions if it is a knot obtained from a trivial $(m+1)$-component link by doing band surgery along $m$ bands. More precisely, it has the form

$$
S_{0}^{1} \cup S_{1}^{1} \cup \cdots \cup S_{m}^{1} \cup \bigcup_{i=1}^{m} f_{i}(\partial I \times I)-\operatorname{int}\left(\bigcup_{i=1}^{m} f_{i}(I \times \partial I)\right),
$$


where $S_{0}^{1} \cup S_{1}^{1} \cup \cdots \cup S_{m}^{1}$ is a trivial link of $m$ components and $f_{i}: I \times I \rightarrow S^{3}$ $(i=1,2, \ldots, m)$ are disjoint embeddings such that

$$
f_{i}(I \times \partial I) \cup S_{j}= \begin{cases}f_{i}(I, 0) & \text { if } j=0 ; \\ f_{i}(I, 1) & \text { if } j=i ; \\ \emptyset & \text { if otherwise }\end{cases}
$$

By a ribbon knot we mean a ribbon knot of $m$-fusions for some $m$; see [16,27]. The least number of such $m$ is the ribbon-fusion number of $K$, which we denote by $\operatorname{rf}(K)$.

Remark 4.1. In $[3,22,26]$ the ribbon-fusion number is called the ribbon number.

If $K$ and $K^{\prime}$ are ribbon knots, then it is easy to see

$$
\operatorname{rf}\left(K \# K^{\prime}\right) \leq \operatorname{rf}(K)+\operatorname{rf}\left(K^{\prime}\right) .
$$

Also, for any $n$-bridge knot $K$, the connected sum of $K$ and its mirror image $K$ !, $K \# K$ ! is a ribbon knot (cf. [23, 8E30]), which satisfies

$$
\operatorname{rf}(K \# K !) \leq n-1 .
$$

Bleiler and Eudave-Muñoz [3] have shown a composite knot with ribbon-fusion number one has a summand that is two-bridge. Then Tanaka [26] proved that there exist composite ribbon-fusion number one knots with arbitrarily large bridge numbers.

The Nakanishi index of a knot $K$, denoted by $m(K)$, is the minimum size among all square Alexander matrix of $K$, provided that $m(K)=0$ if and only if an Alexander matrix of $K$ is equivalent to the $1 \times 1$ matrix with entry 1 as presentation matrices; see [11, p. 72]. Then Makoto Sakuma has given a lower bound of the ribbon-fusion number using the Nakanishi index of a knot [22, Proposition 2].

Proposition 4.2. For a ribbon knot $K$,

$$
\operatorname{rf}(K) \geq m(K) / 2 \text {. }
$$

As applications of Theorems 2.2 and 3.1 we give other lower bounds for the ribbon-fusion number.

Theorem 4.3. If $\operatorname{rf}(K)=n$, then

$$
V(K ; \omega) \in\left\{1, \pm(i \sqrt{3})^{k}, 3^{n} \mid k=1,2, \ldots, 2 n-1\right\} .
$$

In particular, if $K$ is a ribbon knot with $V(K ; \omega)=-3^{n}$, then $\operatorname{rf}(K)>n$.

Proof. We use induction on $n$. If $\operatorname{rf}(K)=1$, then $K$ is obtained from the trivial 2component link $U^{2}$ by a band surgery. Thus since $V\left(U^{2} ; \omega\right)=-\sqrt{3}$, by Corollary 2.3 we obtain Eq. (4.6) with $n=1$. 
Suppose that Eq. (4.6) holds for $n=j$. If $\operatorname{rf}(K)=j+1$, then $K$ is obtained from the split union of a knot $K^{\prime}$ with $\operatorname{rf}\left(K^{\prime}\right)=j$ and the trivial knot, $K^{\prime} \sqcup U$, by a band surgery. Then since $V\left(K^{\prime} \sqcup U ; \omega\right)=-\sqrt{3} V\left(K^{\prime} ; \omega\right)$, by Theorem 2.2 we have $V(K ; \omega) / V\left(K^{\prime} ; \omega\right) \in\{1, \pm i \sqrt{3}, 3\}$. Hence we obtain Eq. (4.6) with $n=j+1$.

Theorem 4.4. If $\operatorname{rf}(K)=n$, then

$$
\rho(K) \in\left\{1, \pm \sqrt{5}^{k}, 5^{n} \mid k=1,2, \ldots, 2 n-1\right\} .
$$

In particular, if $K$ is a ribbon knot with $\rho(K)=-5^{n}$, then $\operatorname{rf}(K)>n$.

Proof. We use induction on $n$. If $\operatorname{rf}(K)=1$, then $K$ is obtained from the trivial 2-component link $U^{2}$ by a band surgery. Thus since $\rho\left(U^{2}\right)=\sqrt{5}$, by Corollary 3.2 we obtain Eq. (4.7) with $n=1$.

Suppose that Eq. (4.7) holds for $n=j$. If $\operatorname{rf}(K)=j+1$, then $K$ is obtained from the split union of a $\operatorname{knot} K^{\prime}$ with $\operatorname{rf}\left(K^{\prime}\right)=j$ and the trivial knot, $K^{\prime} \sqcup U$, by a band surgery. Then by Theorem 3.1 we have $\rho(K) / \rho\left(K^{\prime} \sqcup U\right) \in\left\{ \pm 1, \sqrt{5}^{ \pm 1}\right\}$. Since $Q\left(U^{2} ; z\right)=2 z^{-1}-1$ and $\rho\left(U^{2}\right)=\sqrt{5}, \rho\left(K^{\prime} \sqcup U\right)=\rho\left(K^{\prime}\right) \rho\left(U^{2}\right)=\sqrt{5} \rho\left(K^{\prime}\right)$, and so we have $\rho(K) / \rho\left(K^{\prime}\right) \in\{1, \pm \sqrt{5}, 5\}$. Hence we obtain Eq. (4.7) with $n=j+1$.

Theorems 4.3 and 4.4 immediately imply:

Corollary 4.5. If a knot $K$ satisfies either $V(K ; \omega)=-1$ or $\rho(K)=-1$, then $K$ is not a ribbon knot.

We denote the connected sum of $n$ copies of a knot $K$ by $\stackrel{n}{\#} K$.

Example 4.6. Let $J_{r, s}$ be the connected sum of $r$ copies of the knot $6_{1}$ and $s$ copies of its mirror image $6{ }_{1}$ !. Suppose that $r \geq s$. Then putting $J_{r, s}=$

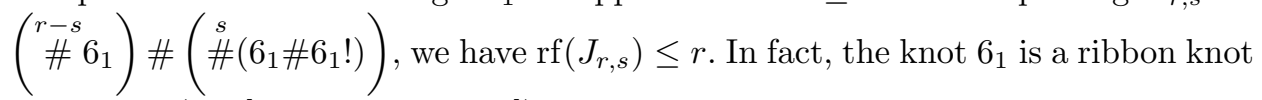
of 1 -fusion (see [11, Appendix F.5]), and also the connected sum $6_{1} \# 6_{1}$ ! is a ribbon knot of 1-fusion since $6_{1}$ is a 2-bridge knot. On the other hand, by Proposition 4.2, $\operatorname{rf}\left(J_{r, s}\right) \geq(r+s) / 2$. Let us consider the case $s=r-2$. Since $V\left(6_{1} ; \omega\right)=i \sqrt{3}$, $V\left(6_{1} ; \omega\right)=-i \sqrt{3}$ (cf. [12, Table 3.1]), we have $V\left(J_{r, r-2} ; \omega\right)=-3^{r-1}$. Thus by Theorem $4.3 \operatorname{rf}\left(J_{r, r-2}\right) \geq r$, and so $\operatorname{rf}\left(J_{r, r-2}\right)=r$, which cannot be deduced from Proposition 4.2.

Example 4.7. Let $K_{n}$ be the connected sum of $n$ copies of the knot $8_{8},(n-1)$ copies of the knot 88 !, and the knot $8_{9}$;

$$
K_{n}=8_{9} \# 8_{8} \#\left(\stackrel{n-1}{\#}\left(8_{8} \# 8_{8} !\right)\right)
$$

Then we have $\operatorname{rf}\left(K_{n}\right)=n+1$. In fact, the knots $8_{8}$ and $8_{9}$ are ribbon knots of 1-fusion (see [11, Appendix F.5]), and the connected sum $8_{8} \# 8_{8}$ ! is also a ribbon knot of 1 -fusion since $8_{8}$ is a 2-bridge knot. Thus $\operatorname{rf}\left(K_{n}\right) \leq n+1$. On the other hand, 
$\rho\left(8_{8}\right)=\sqrt{5}, \rho\left(8_{9}\right)=-\sqrt{5}\left(\right.$ cf. $[4$, Table] $)$ and so $\rho\left(K_{n}\right)=-5^{n}$. Thus by Theorem 4.4 $\operatorname{rf}\left(K_{n}\right)>n$. Note that using Proposition 4.2 , we only have $\operatorname{rf}\left(K_{n}\right) \geq n$.

\section{Band Surgery from a $(2,2 n)$ Torus Link to a $(2 n+1)$-Crossing Knot}

The motivation of this section is the study of Bath, Sherratt, and Colloms [1] of a DNA site-specific recombination; they showed that the action of the Xer site-specific recombination at $p s i$ site is the change from a $(2,2 n)$ torus link to a $(2 n+1)$-crossing knot by a band surgery. So characterizing such change is an important problem. In this section, we consider a problem whether a given knot with $(2 n+1)$ crossings is related to a $(2,2 n)$ torus link or not by a band surgery. Also, DNA knots or links are mainly of 2 bridge, so we consider this problem for 2-bridge knots with 7 or 9 crossings. Applying Corollary 2.3 or Corollary 3.2 we can conclude that some knot cannot be related with a $(2,2 n)$ torus link by a band surgery.

\subsection{Torus links}

First, we calculate some values for torus links needed to apply Eq. (2.2) and Corollaries 2.3 and 3.2. For a positive integer $m$, we denote by $T_{m}$ the oriented torus knot or link of type $(2, m)$ with $m$ crossings as shown in Fig. 4.

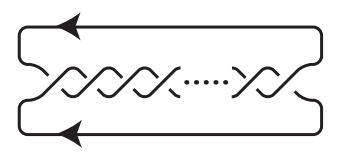

Fig. 4. The oriented torus knot or link of type $(2, m), T_{m}$.

If $m$ is even, then $T_{m}$ is a 2-component link. We denote by $T_{2 n}^{\prime}$ the oriented torus link obtained from $T_{2 n}$ by reversing the orientation of one component, and $T_{2 n}$ !, $T_{2 n}^{\prime}$ ! the mirror images of $T_{2 n}, T_{2 n}^{\prime}$, respectively. Fig. 5 shows torus links $T_{6}$, $T_{6}^{\prime}, T_{6} !, T_{6}^{\prime} !$

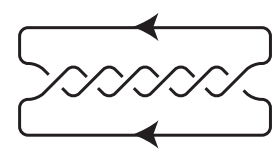

$T_{6}$

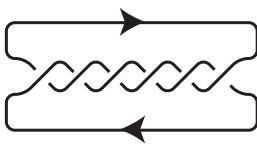

$T_{6}^{\prime}$

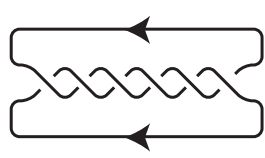

$T_{6}$ !

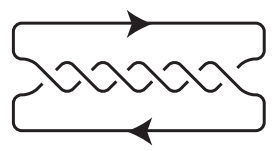

$T_{6}^{\prime}$ !

Fig. 5. Oriented torus links of type $(2,6)$. 
Lemma 5.1. The torus links $T_{2 n}, T_{2 n}^{\prime}, T_{2 n}$ !, $T_{2 n}^{\prime}$ ! have the linking numbers, the signatures, and the values of the Jones polynomials at $t=\omega$ as in Table 1.

Table 1. The linking number, the signature, and the Jones polynomial at $t=\omega$ of the torus links of type $(2,2 n)$.

\begin{tabular}{lrrrrrrrr}
\hline$L$ & $\operatorname{lk}(L)$ & $\sigma(L)$ & \multicolumn{5}{c}{$V(L ; \omega)$} & $(\bmod 6)$ \\
& & & $n \equiv 0$ & $n \equiv 1$ & $n \equiv 2$ & $n \equiv 3$ & $n \equiv 4$ & $n \equiv 5$ \\
\hline$T_{2 n}$ & $-n$ & $2 n-1$ & $-\sqrt{3}$ & $i$ & $i$ & $\sqrt{3}$ & $-i$ & $-i$ \\
$T_{2 n}^{\prime}$ & $n$ & -1 & $-\sqrt{3}$ & $-i$ & $i$ & $-\sqrt{3}$ & $-i$ & $i$ \\
$T_{2 n} !$ & $n$ & $-2 n+1$ & $-\sqrt{3}$ & $-i$ & $-i$ & $\sqrt{3}$ & $i$ & $i$ \\
$T_{2 n}^{\prime} !$ & $-n$ & 1 & $-\sqrt{3}$ & $i$ & $-i$ & $-\sqrt{3}$ & $i$ & $-i$ \\
\hline
\end{tabular}

Proof. The signatures of $T_{2 n}$ and $T_{2 n}$ ! are given in [5, Theorem 5.2]; cf. [21, Theorem 7.5.1]. We obtain the signatures of $T_{2 n}^{\prime}$ and $T_{2 n}^{\prime}$ ! by the following formula due to Murasugi [20, Theorem 1]:

$$
\sigma\left(L^{\prime}\right)=\sigma(L)+2 \operatorname{lk}(L)
$$

where $L$ is an oriented 2-component link with linking number $1 \mathrm{k}(L)$ and $L^{\prime}$ a link obtained from $L$ by reversing the orientation of one component

Now we consider the Jones polynomial of $T_{n}^{\prime}$. Since $\left(T_{2 n}^{\prime}, T_{2 n-2}^{\prime}, U\right)$ is a skein triple, from Eq. (2.4) we have

$$
t^{-1} V\left(T_{2 n}^{\prime} ; t\right)-t V\left(T_{2 n-2}^{\prime} ; t\right)=t^{1 / 2}-t^{-1 / 2} .
$$

Then

$$
\begin{aligned}
V\left(T_{2 n}^{\prime} ; t\right)-\mu^{-1} & =t^{2}\left(V\left(T_{2 n-2}^{\prime} ; t\right)-\mu^{-1}\right) \\
& =t^{2 n}\left(V\left(T_{0}^{\prime} ; t\right)-\mu^{-1}\right) \\
& =t^{2 n}\left(\mu-\mu^{-1}\right)
\end{aligned}
$$

where $\mu=V\left(U^{2} ; t\right)=-t^{1 / 2}-t^{-1 / 2}$. Then

$$
\begin{aligned}
V\left(T_{2 n}^{\prime} ; \omega\right) & =-\sqrt{3}^{-1}+\omega^{2 n}\left(-\sqrt{3}+\sqrt{3}^{-1}\right) \\
& =\left\{\begin{array}{lll}
-\sqrt{3} & \text { if } n \equiv 0 & (\bmod 3) ; \\
-i & \text { if } n \equiv 1 & (\bmod 3) ; \\
i & \text { if } n \equiv 2 & (\bmod 3) .
\end{array}\right.
\end{aligned}
$$

Since $V\left(T_{2 n}^{\prime} ! ; t\right)=V\left(T_{2 n}^{\prime} ; t^{-1}\right)\left[7\right.$, Theorem 3] and $\omega^{-1}=\bar{\omega}$, the complex conjugate of $\omega$, we have $V\left(T_{2 n}^{\prime} ! ; \omega\right)=\overline{V\left(T_{2 n}^{\prime} ; \omega\right)}$. Since $V\left(T_{2 n} ; t\right)=t^{-3 n} V\left(T_{2 n}^{\prime} ; t\right)$ $[13,18]$, we have $V\left(T_{2 n} ; \omega\right)=(-1)^{n} V\left(T_{2 n}^{\prime} ; \omega\right)$. Similarly, we have $V\left(T_{2 n} ! ; \omega\right)=$ $(-1)^{n} V\left(T_{2 n}^{\prime} ! ; \omega\right)$. Then we obtain Table 1 . 
Let $\rho_{m}=\rho\left(T_{m}\right)$. Then we have the following.

\section{Lemma 5.2 .}

$$
\rho_{m}= \begin{cases}\sqrt{5} & \text { if } m \equiv 0 \quad(\bmod 5) ; \\ 1 & \text { if } m \equiv 1,4 \quad(\bmod 5) \\ -1 & \text { if } m \equiv 2,3 \quad(\bmod 5)\end{cases}
$$

Proof. From an unoriented skein quadruple $\left(T_{m+1}, T_{m-1}, T_{m}, U^{2}\right)$, where $U^{2}$ is the trivial 2-component link, by Eq. (3.2) we have

$$
Q\left(T_{m+1} ; z\right)+Q\left(T_{m-1} ; z\right)=z\left(Q\left(T_{m} ; z\right)+Q\left(U^{2} ; z\right)\right) .
$$

Since $\rho\left(U^{2}\right)=\sqrt{5}$, we have

$$
\rho_{m+1}+\rho_{m-1}=\frac{\sqrt{5}-1}{2}\left(\rho_{m}+\sqrt{5}\right) .
$$

Using $\rho_{0}=\sqrt{5}, \rho_{1}=1$, we obtain Eq. (5.5).

Combining Theorem 3.1 and Lemma 5.2, we obtain immediately the following.

Corollary 5.3. Suppose that a knot $K$ is obtained from a torus link of type $(2,2 n)$ by a band surgery. Then

$$
\rho(K) \in \begin{cases}\{1, \pm \sqrt{5}, 5\} & \text { if } n \equiv 0 \quad(\bmod 5) ; \\ \{ \pm 1,-\sqrt{5}\} & \text { if } n \equiv 1,4 \quad(\bmod 5) ; \\ \{ \pm 1, \sqrt{5}\} & \text { if } n \equiv 2,3 \quad(\bmod 5) .\end{cases}
$$

\subsection{7-crossing 2-bridge knots}

We consider the problem whether a 7 -crossing 2 -bridge knot is related to a $(2,6)$ torus link or not by a band surgery. According to Shimokawa, the knots $7_{1}, 7_{2}, 7_{4}$ are obtained from a $(2,6)$ torus link.

First, we consider applying Corollary 2.3. The $\gamma$-values of $(2,6)$ torus links are $\pm i \sqrt{3}$ from Table 1 . Then we can apply Corollary 2.3 for a knot $K$ with $V(K ; \omega)=$ \pm 1 . Note that the determinant of such a knot is $\not \equiv 0(\bmod 3)$; see Eq. (2.5). Since the determinants of the knots $7_{3}, 7_{5}, 7_{6}, 7_{7}$ are $13,17,19,21$, respectively, we should test this method except for the knot $7_{7}$. Then for the knot $7_{3}$ and $7_{6}$ we can obtain the result.

Proposition 5.4. The knots $7_{3}$ and $7_{6}$ cannot be obtained from a $(2,6)$ torus link by a band surgery. 
Proof. Suppose that the knot $7_{6}$ is related with a $(2,6)$ torus link by a band surgery. Since $\sigma\left(7_{6}\right)=2$ (cf. [12, Table 8.1]), by Eq. (2.2) $T_{6}^{\prime}$ ! should be such a torus link. From Table $1 V\left(T_{6}^{\prime} ! ; \omega\right)=-\sqrt{3}$, and so by Corollary $2.3 V\left(7_{6} ; \omega\right) \in$ $\{1, \pm i \sqrt{3}, 3\}$, which is a contradiction since $V\left(7_{6} ; \omega\right)=-1$ (cf. [12, Table 3.1]).

For the knot $7_{3}$, the proof is similar. Since $\sigma\left(7_{3}\right)=-4$, we have to consider the link $T_{6}$ ! Suppose that $7_{3}$ is related with $T_{6}$ ! by a band surgery. Since $V\left(T_{6} ! ; \omega\right)=\sqrt{3}$, $V\left(7_{3} ; \omega\right) \in\{-1, \pm i \sqrt{3},-3\}$, which is a contradiction since $V\left(7_{3} ; \omega\right)=1$.

Remark 5.5. Kawauchi has proved that $7_{3}$ and $7_{7}$ cannot be obtained from a $(2,6)$ torus link by a band surgery using the Alexander invariants. Also, Darcy, Ishihara, Shimokawa have given a characterization of band surgery for the knots $7_{2}$ and $7_{4}$. So the question whether the knot $7_{5}$, whose signature is -4 , is related by a band surgery to a $(2,6)$ torus link $T_{6}$ ! or not remains open.

For a 7 crossing knot, we cannot apply Corollary 5.3. In fact, in order to apply Corollary 5.3 the knot should satisfy $\rho(K)=-\sqrt{5}$. Then the determinant of such a knot is $\equiv 0(\bmod 5)$; see Eq. (3.3).

\subsection{9-crossing 2-bridge knots}

We consider the problem whether a 9-crossing 2-bridge knot is related to a $(2,8)$ torus link or not by a band surgery. Since $(2,8)$ torus links have signatures \pm 1 or \pm 7 (Table 1 ), a knot with signature \pm 4 is never related to $(2,8)$ torus links by a band surgery by Eq. (2.2). The following knots have signature $\pm 4: 9_{4}, 9_{7}, 9_{10}, 9_{11}$, $9_{13}, 9_{18}, 9_{20}, 9_{23}$; see [11, Appendix F.3]. Also, it is easy to see that the knots $9_{1}$, $9_{2}$ are related to a $(2,8)$ torus link by a band surgery.

First, we consider applying Corollary 2.3. The $\gamma$-values of $(2,8)$ torus links are \pm 1 (Table 1 ), and so we can apply Corollary 2.3 for a $\operatorname{knot} K$ with $V(K ; \omega)= \pm i \sqrt{3}$. Note that the determinant of such a knot is $\equiv 0(\bmod 3)$; see Eq. $(2.5)$. Thus we apply this method for the knots $9_{6}, 9_{15}, 9_{17}$, whose determinants are $27,39,39$, respectively.

Proposition 5.6. The knots $9_{15}$ and $9_{17}$ cannot be obtained from a $(2,8)$ torus link by a band surgery.

Proof. The proof is similar to that of Proposition 5.4. We list the necessary data:

$$
\begin{array}{ll}
\sigma\left(9_{15}\right)=-2, & V\left(9_{15} ; \omega\right)=-i \sqrt{3} \\
\sigma\left(9_{17}\right)=2, & V\left(9_{17} ; \omega\right)=i \sqrt{3} .
\end{array}
$$

Next, we consider applying Corollary 5.3. We can apply Corollary 2.3 for a knot $K$ with $\rho(K)=-\sqrt{5}$. Note that the determinant of such a knot is $\equiv 0(\bmod 5)$. Thus we apply this method for the knots $9_{6}, 9_{15}, 9_{17}$. 
Proposition 5.7. The knot $9_{31}$ cannot be obtained from a $(2,8)$ torus link by a band surgery.

Proof. Suppose that the knot $9_{31}$ is related with a $(2,8)$ torus link by a band surgery. By Lemma $5.2 \rho\left(T_{8}\right)=-1$, and so by Corollary $3.2 \rho\left(9_{31}\right) \in\{ \pm 1,-\sqrt{5}\}$, which is a contradiction since $\rho\left(9_{31}\right)=\sqrt{5}$. (Note that $Q\left(9_{31}\right)=-7+12 z+36 z^{2}-$ $22 z^{3}-58 z^{4}-4 z^{5}+28 z^{7}+14 z^{8}+2 z^{9}$, which is obtained from the Kauffman $F$ polynomial listed in [11, Appendix F.6].)

For the following 9 crossing 2-bridge knots we cannot decide whether they are related to a $(2,8)$ torus link or not by a band surgery using our method:

$$
9_{k}, \quad k=3,5,6,8,9,12,14,19,21,26,27 .
$$

\section{Acknowledgments}

The author would like to thank Professor Koya Shimokawa for introducing the problem in Sect. 5. This work was partially supported by Grant-in-Aid for Scientific Research (C) (No. 21540092), Japan Society for the Promotion of Science.

\section{References}

[1] J. Bath, D. J. Sherratt and S. D. Colloms, Topology of Xer recombination on catenanes produced by lambda integrase, J. Mol. Biol. 289 (1999) 873-883.

[2] J. S. Birman and T. Kanenobu, Jones' braid-plat formula and a new surgery triple, Proc. Amer. Math. Soc. 102 (1988) 687-695.

[3] S. A. Bleiler and M. Eudave-Muñoz, Composite ribbon number one knots have twobridge summands, Trans. Amer. Math. Soc. 321 (1990) 231-243.

[4] R. D. Brandt, W. B. R. Lickorish and K. Millett, A polynomial invariant for unoriented knots and links, Inv. Math. 84 (1986) 563-573.

[5] C. McA. Gordon, R. A. Litherland and K. Murasugi, Signatures of covering links, Canad. J. Math. 33 (1981) 381-415.

[6] C. F. Ho, A polynomial invariant for knots and links - preliminary report, Abstracts Amer. Math. Soc. 6 (1985) 300

[7] V. F. R. Jones, A polynomial invariant for knots via von Neumann algebra, Bull. Amer. Math. Soc. 12 (1985) 103-111.

[8] V. F. R. Jones, Hecke algebra representations of braid groups and link polynomials, Ann. Math. 126 (1987) 335-388.

[9] V. F. R. Jones, On a certain value of the Kauffman polynomial, Comm. Math. Phys. (1989) 103-111.

[10] T. Kanenobu and Y. Miyazawa, H(2)-unknotting number of a knot, Communications in Mathematical Research (to appear).

[11] A. Kawauchi, A Survey of Knot Theory, (Birkhäuser Verlag, Berlin, 1996).

[12] W. B. R. Lickorish, An introduction to Knot Theory, Graduate Texts in Math., 175 (Springer-Verlag, New York, 1997).

[13] W. B. R. Lickorish and K. C. Millett, The reversing formula for the Jones polynomial, Pacific J. Math. 124 (1986) 173-176. 
[14] W. B. R. Lickorish and K. C. Millett, Some evaluations of link polynomials, Comment. Math. Helv. 61 (1986), 349-359.

[15] A. S. Lipson, An evaluation of a link polynomial, Math. Proc. Camb. Phil. Soc. 100 (1986) 361-364.

[16] Y. Marumoto, On ribbon 2-knots of 1-fusion, Math. Sem. Notes, Kobe Univ. 5 (1977) $59-68$.

[17] Y. Miyazawa, Talk at the conference "The 5th East Asian School of Knots and Related Topic" (Gyeongju, Korea, 2009).

[18] H. R. Morton, The Jones polynomial for unoriented links, Quart. J. Math. Oxford 37 (1986) 55-60.

[19] K. Murasugi, On the certain numerical invariant of links, Trans. Amer. Math. Soc. 117 (1965) 387-422.

[20] K. Murasugi, On the signature of links, Topology 9 (1970) 283-298.

[21] K. Murasugi, Knot Theory and Its Applications (Birkhäuser, 1996).

[22] Y. Nakanishi and Y. Nakagawa, On ribbon knots, Math. Sem. Notes, Kobe Univ. 10 (1982) 423-430.

[23] D. Rolfsen, Knots and Links, (AMS Chelsea Pub. 2003).

[24] Y. Rong, The Kauffman polynomial and the two-fold cover of a link, Indiana Univ. Math. J. 40 (1991) 321-331.

[25] A. Stoimenow, Polynomial values, the linking form and unknotting numbers, Math. Res. Lett. 11 (2004) 755-769.

[26] T. Tanaka, On bridge numbers of composite ribbon knots, J. Knot Theory Ramifications 9 (2000) 423-430.

[27] T. Yanagawa, On ribbon 2-knots. I. The 3-manifold bounded by the 2-knots. Osaka J. Math. 6 (1969) 447-464. 